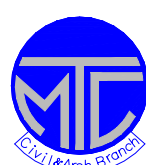

ICCAE

Military Technical College

Kobry Elkobbah,

Cairo, Egypt 6hㅡ International Conference

On Civil \& Architecture

Engineering

\title{
Numerical Simulation Capabilities for Ground Shock Prediction due to Subsurface Explosion
}

\author{
Louay, S. Mohamed ${ }^{1}$, Hassan, M. Farag ${ }^{2}$, Mohamed, N. Fayed ${ }^{3}$ \\ Abou Zied, M. Tarkhan ${ }^{4}$
}

\begin{abstract}
The ground shock produced by subsurface explosion near buried structures generally represents the dominant threat to these facilities. Due to various constraints, pertinent experimental data are extremely scarce. Adequately detailed numerical simulation thus becomes a desirable alternative. The use of commercially available codes, which are capable of solving non-linear dynamic problems, validated against proven prediction techniques and experimental data, can provide a cost-effective means of examining the highly dynamic processes involved in the subsurface explosion and ground shock propagation. This paper sets out to examine the potential of numerical analysis for investigating ground shock phenomena from underground explosion in different sites. The finite element code AUTODYN, which is available in 2-dimensional (2D) and 3-dimensional (3D) formats was employed. The study presents best material models necessary to produce good correlation with free filed resultants obtained from filed tests and CONWEP, an established experimentally-based prediction technique. The validated numerical model is then used to study the ground shock propagation in three different rock types; hard, moderate and soft rocks.
\end{abstract}

\section{Keywords}

Subsurface explosion, Ground shock propagation, Numerical simulation

1- Ph.D, Candidate, Syria

2- Ph.D, Egyptian Armed Forces.

3- Professor, Ain Shams University, Egypt

4- Ass. Professor, Helwan University, Egypt 


\section{Introduction}

The ground shock produced by subsurface blast near buried structures generally represents the dominant threat to these facilities. Stresses from buried bursts are usually greater in magnitude and have much longer duration than corresponding bursts in air [1]. When a charge is detonated below the ground surface, a part of the released energy gets transmitted directly into the surrounding soil mass in the form of seismic stress waves. All of the major parameters, peak stress, peak particle velocity (PPV), peak particle acceleration (PPA), peak particle displacement (PPD) and specific impulse, reduce with the distance from the events. The rate of attenuation with range is governed by soil type and characterized by the attenuation coefficient $n$. A large number of trials have resulted in empirical relationships which permit predictions of the ground shock parameters [2].

Since soil damage and stress wave propagation from underground explosion in soil mass are highly dependent on material properties, data obtained from one site might not be directly used to another site. Investigation of underground blast and its effects by scaled and full size experiments in every site is very expensive. Thus, a reliable numerical model validated against filed measured data, is a cost-effective means for examining the highly dynamic and nonlinear process of blast-induced stress wave propagation in engineering.

Besides the problem of high computational cost, the difficulties associated with modeling the dynamic interaction between the explosion and the soil mass is a major barrier. As the stress condition varies dramatically in the near field of the charge, it is very difficult to model the behavior of soil in this region. A major difficulty in the simulation process of subsurface blast is the formation of the crater by the explosion. The explosion product expands enormously. The soil in the vicinity of the charge undergoes large deformation. Large deformations can result in a severe mesh distortion and hence reduce the accuracy and the time step sharply [3].

\section{Proposal numerical model}

In the numerical simulations, the partial differential equations governing the basic physical principles of conservation of mass, momentum, and energy are employed. The equations to be solved are both time and space-dependent and nonlinear in nature. These equations, together with equations of state (EOS) and constitutive models describing material behavior and a set of initial and boundary conditions, define the complete system for blast simulations [4].

The solution over the time domain can be achieved by an explicit method. In the explicit method, the solution at a given point in time is explicitly expressed as a function of the system variables and parameters, with no requirement for stiffness and mass matrices. Thus the computing cost at each time step is low but may also require numerous time steps for a complete solution.

There are two major ways of describing the solution over the space domain, based on the relative movement between the material particles and the mesh: one is the Eulerian description, while the other is Lagrangian description [4]. In the Eulerian description, the mesh is fixed in space and different material particles move through it. In the Lagrangian 
description, the mesh and the material particles coincide. The Eulerian description is suited for situations where the mesh may be highly distorted; but modeling of the material boundary conditions such as slippage and contact surface is very difficult. The Lagrangian description is more suitable for situations where the deformation is not large but the effects of interface and free boundaries are significant. This concept leads to a requirement for a numerical technique that allows both Eulerian and Lagrangian solutions in a single simulation with coupling between the different techniques in space and time. Such an approach, wherein different methods may be applied within a single numerical analysis, can provide the "best" solution in terms of accuracy and efficiency.

In the problem under investigation, the two descriptions are used in a single analysis. The Eulerian description is used for detonation of explosive material, while the Lagrangian description is suitable for representation of soil material location and ground shock propagation. The AUTODYN Auler-Lagrange coupling facility is employed in the problem. To overcome the highly Lagrangian mesh distortion in the vicinity of the charge, the erosion criteria is used with suitable values [4].

\subsection{Material models}

There are two kinds of materials involved in the problem under investigation, namely the soil mass (rock, clay, sand), and the high energy charge. The models proposed to simulate the behavior of these materials under shock loading are as follows:

\section{Rock modeling}

Since rock has low porous (negligible compression) it is useful to apply a linear relationship between hydrostatic pressure and density (linear EOS). The RHT constitutive model is a new model for general brittle materials, developed by Riedel, Hiermaier and Thoma and implemented into AUTODYN [4,5]. This model contains many features common to various similar constitutive models such as: pressure hardening, strain hardening, strain rate hardening, and cumulative damage (strain softening).

The material model uses three strength surfaces: an elastic limit surface, a failure surface and the remaining strength surface for the crushed material. Fig. (1) shows these strength surfaces. More about this material model can be found in $[4,5]$.

\section{Clay modeling}

Saturated clay is modeled with the elasto-plastic hydro material model. For saturated clays the water content has a profound influence on ground shock propagation. When the saturation approaches one hundred percent, peak stresses and accelerations similar to shock wave propagation in free water have been observed [1], therefore, the shock EOS is used [4].

The Von Mises yield surface is used as a strength model [4]. A hydro tensile failure limit with a small negative pressure value is defined.

\section{Sand modeling}

Since sand is a granular material, it is necessary to adopt a granular material model with Compaction EOS when sand is subjected to blast loads. Laine and Sandvik [6] had 
performed four tri-axial cylindrical tests and three tri-axial shear tests to derive the mechanical properties of sand utilized for this model.

$A$ hydro tensile failure is also used for the sand, and the hydro tensile limit is set to be $\mathrm{p}$ min $=-1 \mathrm{e}^{-3} \mathrm{~Pa}$ as the failure criterion [6]. This means that when the $\mathrm{p}$ min is reached, the cell is not allowed to resist shear stresses.

\section{Explosion modeling}

The high explosive blast wave modeling facility in AUTODYN-2D is a capability that has only recently been exploited on the so-called remapping function. Remapping allows taking the solution of 2D analysis and impose it upon a selected region, of a 3D model where an extra physical dimension can be modeled. This procedure not only reduces the time required for a calculation but also increases its accuracy due to the fine $2 \mathrm{D}$ mesh resolution in the initial high explosive detonation and expansion phases. The explosive material at start time is modeled by the "Jones - Wilkins - Lee" (JWL) EOS that models the pressure generated by chemical energy in an explosive, it can be written in the form [4]

$$
P=C_{1}\left(1-\frac{\omega}{r_{1} v}\right) e^{-r_{1} v}+C_{2}\left(1-\frac{\omega}{r_{2} v}\right) e^{-r_{2} v}+\frac{\omega e}{v}
$$

where $C_{1}, C_{2}, r_{1}$ and $r_{2}$ are constants and $e, \omega$ and $v$ are the internal energy, adiabatic constant and specific volume respectively, and its values for many common explosives have been determined from dynamic experiments and are available in AUTODYN material library [4].

From this equation it can be shown that at large expansion ratios the first and second terms on the right hand side of the equation become negligible and hence the behavior of the explosive tends towards that of an ideal gas. Therefore, at large expansion ratios, where the explosive has expanded by a factor of approximately 10 from its original volume, it is valid to switch the EOS for a high explosive from JWL to ideal gas.

\subsection{Boundaries and interactions modeling}

Two symmetry plans are used in the finite element model; they intersect at the center of gravity of the charge. Transmit boundary conditions is applied to limit the reflection of stress waves from numerical boundaries of soil mass and free boundary surface is applied at the top (ground surface). The explosive-soil interaction is simulated using 3D Euler-Lagrange coupling facility in AUTODYN [4].

\section{Numerical applications}

Three numerical applications are presented to demonstrate the implementation of proposal numerical model to estimate the ground shock propagation in different sits.

\subsection{First application}

The application is one of the filed tests conducted by Hayes according to ref. [7] in nearly saturated clay site. The assumed soil properties are: the initial density is $1900 \mathrm{~kg} / \mathrm{m}^{3}$, the shear modulus is $1.01 \mathrm{GPa}$, and the yield stress is $400 \mathrm{KPa}$. The explosive source is a 
cylindrical charge of $7 \mathrm{~kg} \mathrm{C}$, its diameter is $90 \mathrm{~mm}$ and it has $686 \mathrm{~mm}$ long. In these experiments, accelerometers and pressure sensors were placed in the soil at various ranges from the charge to provide free-field stress and motion measurements. Accelerometer records are integrated once to obtain free-field velocities and again to obtain displacements. A cross section through field test is shown in Figure (2). Data were only reported for $12 \mathrm{msec}$.

\section{Numerical results and analysis}

The proposed numerical model is used to simulate the filed blasting test in nearly saturated clay medium. The attenuation of pressure with time and distance is shown in Figure (3). Figure (4) shows comparison of pressure time history at $1.5 \mathrm{~m}$ from charge center obtained from the numerical analysis with those obtained from filed test at the same point. It shows that the arrival time of the blast wave and its peak agree well with the field test.

Figure (5) shows the comparison of numerical results with those obtained from CONWEP [8], an established experimentally-based prediction technique. The comparison demonstrates that, the peak stresses obtained from CONWEP are matching the numerical results for ranges more than $2 \mathrm{~m}$ reasonably well, while for ranges less than $2 \mathrm{~m}$, CONWEP overpredicts the peak stresses. This is may be because of the difficulty of placing the gages in soil closes to the high explosive charges. Summary of the comparison of results obtained from numerical analysis with those obtained from experimental, DYNA-2D and CONWEP are presented in Table (1).

\subsection{Second application}

This example sets out to examine the potential of the proposed model for ground shock prediction resulting from subsurface explosion in sandy site. However, in the absence of filed test results, it is useful to compare the numerical results with those obtained from proven prediction techniques such as CONWEP [8]. The case study demonstrated in the first application is applied in sandy medium. The initial properties chosen for sand are: dry mass density $1671 \mathrm{~kg} / \mathrm{m}^{3}$, shear modulus $150 \mathrm{MPa}$, attenuation coefficient 3, friction angle $35^{\circ}$.

\section{Numerical results}

The attenuation of pressure with time and distance is shown in Figure (6). This Figure also demonstrates the ability of dry sand to absorb blast shock energy. For example, the peak stress at $1.5 \mathrm{~m}$ and $3 \mathrm{~m}$ from the charge center is $1.2 \mathrm{MPa}$ and $0.35 \mathrm{MPa}$ respectively, while in clay site (first application) and at the same ranges peak stresses are $7 \mathrm{MPa}$ and $2 \mathrm{MPa}$. In Figure (7-a), the obtained peak stresses form numerical analysis are compared with the corresponding results obtained from CONWEP. Figure (7-b) shows the comparison between stress time histories obtained from analysis with those obtained from CONWEP results at $3 \mathrm{~m}$ from the explosive charge. It shows that the blast wave form, the arrival time and the peak value obtained from numerical analysis agree well with that obtained from CONWEP.

\subsection{Third numerical application}

A series of filed blasting testes were carried out by Zhuo et al. [9] at the granite site. The cross section of filed layout is shown in Figure (8). More than 100 gages, including 
accelerometers, pressure sensors and strain gages were used for different measurements. The measuring points were placed along two lines on rock surface and in rock mass as shown in Figure (8). Eight testes were carried out with the equivalent TNT charge weight from $5 \mathrm{~kg}$ to $50 \mathrm{~kg}$. In order to simulate a confined explosion, the charge chamber (bottom $5 \mathrm{~m}$ of the charge hole) was covered by 8 concrete blocks with a total weight of $15 \mathrm{t}$ to prevent any uplifting. In each test, explosive was placed at the center of the charge chamber on a wooden stand. Before the blast tests, intensive filed and laboratory investigations had been conducted to study the site-properties. Summary of these investigations are given in Table (2).

\section{Numerical results and analysis}

The proposed numerical model is used to simulate the filed blasting test in the granite mass. The corresponding charge weight is $50 \mathrm{~kg}$ TNT. Obtained velocity and acceleration time histories from numerical simulation in rock mass at $25 \mathrm{~m}$ from charge hole in $\mathrm{Y}$ direction are shown in Figure (9). Figure (10) shows comparison of acceleration time history at $25 \mathrm{~m}$ from charge hole in the $\mathrm{Y}$ direction obtained from the numerical analysis with that measured from filed test and anisotropic numerical model proposed by Hao et al. [10] at the same point. It shows that the arrival time, the wave form and peak value of the simulated stress wave obtained by the proposed model agree better with those of the recorded field results than those obtained from anisotropic model.

\section{Prediction of peak pressure and PPV in rocks}

Probably the most difficult phase of designing a buried structure to resist the effects of conventional weapons is the determination of the loads on the structure. The ground shock resulting from subsurface explosion is expressed in terms of peak stress $\left(P_{0}\right)$ and $P P V$. Generally the free filed stresses (the stresses would be present if the structure was absent) and particle velocities at the point on the structure are computed. These stresses are modified to approximate the effects on the structure and its response. These modified stresses (interface stresses) are applied as the structure loading [2]. The Technical manual TM-5 [1] suggested two functions to estimate these parameters $\left(P_{0}\right.$ and $\left.P P V\right)$ in cohesive soils

Function 1: $P_{0}=f \cdot 15 \cdot(R / \sqrt[3]{W})^{-n_{1}}, \mathrm{MPa}$

Function 2: $P_{0}=f \cdot 22 \cdot(R / \sqrt[3]{W})^{-n_{2}}, \mathrm{MPa}$

Function 1: $P P V=f \cdot 4.62 \cdot(R / \sqrt[3]{W})^{-n_{1}}, \mathrm{~m} / \mathrm{s}$

Function 2: $P P V=f \cdot 7.38 \cdot(R / \sqrt[3]{W})^{-n_{2}}, \mathrm{~m} / \mathrm{s}$

where $n_{1}, n_{2}$ are dimensionless attenuation coefficients. Some typical values of $n$ are given for a range of soils, more detail may be found in refs. [1-3], $f$ is the ground shock coupling factor determined by the depth of burial from ref. [3], $R$ is the distance from the charge center to the point of interest in meters, and $W$ is the explosive weight in kg. These two functions can be looked upon as the upper and lower limits within which $P_{0}$, and $P P V$ in particular type of soil should fall.

Using the proposed examined numerical model for ground shock prediction in granite (third application), three rows of target points are arranged parallel to the ground surface as 
shown in Figure (11). Group-A targets are arranged at depth of $2.7 \mathrm{~m}$ (at the charge gravity center), while Group-B and Group-C targets are located at depth of $1.7 \mathrm{~m}$ and $3.7 \mathrm{~m}$ from the ground surface respectively to capture the "free field" wave propagation in three rock types; hard, moderate and soft rocks. The mechanical properties for three rock types are given in Table (3). The values of strength and moduli are determined from numerous references $[11,12]$.The rock media is assumed to be continuous, isotropic and homogeneous medium. The potential threat corresponds to the fully buried general purpose bombs of type MK-82, MK-84 and M-118. The general characteristics of these bombs are given in Table (4) [1].

In Figure (12), the crater formulation in three rock types corresponding to subsurface explosion of MK-84 general purpose bomb at $1.7 \mathrm{~m}$ from the ground surface is shown. It is clear that in hard rock, the rock mass is ejected away from the blast, while in soft rock the explosion forms a camouflet. This is reasonable because as the rock mass get harder, the blast resistance is higher and the blast energy will go by essential way (to the ground surface). This reflects that, the damage zone is bigger in soft rock mass than in moderate and hard rocks as shown in Figure (13).

\subsection{Proposal formula for ground shock prediction in different rocks}

The recorded numerical results at all target points are used to derive empirical attenuation functions for $P_{0}$ and $P P V$ in different types of rock.

The best fitted empirical attenuation expressions for peak free field pressure $\left(P_{0}\right)$ are given as follows:

Function 1: $P_{0}=8 \cdot f \cdot(R / \sqrt[3]{W})^{-1.313}, \mathrm{MPa}$

Function 2: $P_{0}=12 \cdot f \cdot(R / \sqrt[3]{W})^{-1.227}, \quad \mathrm{MPa}$

where function 1 and function 2 give the upper and lower limits for peak pressure $P_{0}$ according the rock type. It should be noted that the above equations are derived by using the data obtained from numerical analysis with scaled distances in the range from $0.2 \mathrm{~m} \cdot \mathrm{kg}^{-1 / 3}$ to 2 $\mathrm{m} . \mathrm{kg}^{-1 / 3}$.

Figure (13) shows the attenuation of the peak pressure as a function of the scaled distance for the group-A group-B, and Group-C targets in three different types of rock.

The best fitted empirical attenuation relations for $P P V$ in three rock types are given as follows:

$$
\begin{array}{lll}
P P V=1.667 \cdot f \cdot(R / \sqrt[3]{W})^{-1.217}, \mathrm{~m} / \mathrm{s} & \text { in hard rock } \\
P P V=2.061 \cdot f \cdot(R / \sqrt[3]{W})^{-1.284}, \mathrm{~m} / \mathrm{s} & \text { in moderate rock } \\
P P V=3.222 \cdot f \cdot(R / \sqrt[3]{W})^{-1.298}, \mathrm{~m} / \mathrm{s} & \text { in soft rock }
\end{array}
$$

Figure (14) shows the attenuation of the PPV as a function of the scaled distance for the group-A group- $\mathrm{B}$, and Group- $\mathrm{C}$ targets in three rock types.

\section{Conclusions}

Implementing the proposed 3D numerical model in AUTODYN hydrocode, the ground shock propagation resulting from subsurface explosion in different mediums has been simulated. The validity of the model for ground shock prediction has been proven to be 
comparable with an independently conducted field tests and CONWEP. It was found that the ground shock aspects (arrival times, peak stresses, peak particle velocities, peak particle acceleration and the decrease of slope of the shock front in the ground) agree well with field tests and CONWEP.

The numerical results demonstrate the ability of dry sand to absorb the high stress blast energy and it can be used as good absorption filling material for buried structures constructed in dense soils.

Proposed formulae for ground shock prediction in different rock mediums were presented to give upper and lower limits for peak pressure and peak particle velocities.

\section{References}

[1] Technical Manual TM5-885-1, "Fundamentals of Protective Design for Conventional Weapons", Headquarters Department of the Army, Washington, DC, 1986.

[2] Bulson P. S., “Explosive Loading of Engineering Structures”, Londn,1997.

[3] Agardh, L., "Validation of Numerical Simulations of Structural Response via Material Sample Testing and Field Trials”, $3^{\text {rd }}$ Asia-Pacific Conference on Shock \& Impact Loads on Structures, pp.1-14, Singapore, Nov., 1999.

[4] AUTODYN Theory Manuals, Version 4.3, Century Dynamics, Inc.2003 Sam Ramon, USA, 2003.

[5] Riedl W., Thoma K., Hiermaier S., "Numerical Analysis Using a New Macroscopic Concrete Model for Hydrocodes ”, Proceedings of 9th International Symposium of the Effects of Munitions With Structures, 1999.

[6] Laine L. and Sandvik A., "Derivation of Mechanical Properties for Sand", $4^{\text {th }}$ AsiaPacific Conference on Shock \& Impact Loads on Structures, Singapore, pp 361-368, November,2001.

[7] Baylot J. T., "Effect of Soil Flow Changes on Structure Loads", Journal of Structural Engineering, Vol.126, No. 12, December, pp 1434-1441, 2000.

[8] Hyde D.W., "CONWEP-Conventional Weapons Effects Program", US Army Waterways Experiment Station, Vickspurg, MS, 1991.

[9] Zhou Y, Hao H., Wu Y., and Ma G., "Characteristics of Surface Ground Motion Induced by Blasts in Jointed Rock Mass", Soil Dynamics and Earthquake Engineering, Vol. 21, pp. 85-98, 2000.

[10] Hao H., Wu C. and Zhou Y., "Numerical Analysis of Blast-Induced Stress Wave in a Rock Mass with Anisotropic Continuum Damage Models Part1: Equivalent Material Property Approach", Rock Mech. Rock Engng. , Vol. 35 (2), pp. 79-94, 2002.

[11] Richard E., "Introduction To Rock Mechanics”, Goodman University of California John Wiley \& Sons Isbno-471-04129-7, 1980.

[12] Hussien A. Loulou, "Nonlinear Dynamic Analysis of Tunnels Subjected to Explosion”, Ph.D, M.T.C, Cairo, 2005. 
Table (1) Comparison of results at $1.5 \mathrm{~m}$ from the charge center

\begin{tabular}{|l|c|c|c|c|}
\hline Parameter & Field Test & DYNA-2D & CONWEP & Autodyn-3D \\
\hline \hline Peak Stress, MPa & 7 & 7.5 & 7.649 & 6.968 \\
\hline Arrival Time, msec & 1.75 & 1.8 & 3.3 & 1.71 \\
\hline PPV, m/s & 8.7 & 10.5 & 8.051 & 7.628 \\
\hline
\end{tabular}

Table (2) Filed and laboratory investigations from granite site [9]

\begin{tabular}{|l|c|}
\hline Granite properties & value \\
\hline \hline average rock density $\left(\mathrm{kg} / \mathrm{m}^{3}\right)$ & 2610 \\
\hline average uniaxial compressive strength (MPa) & 148 \\
\hline Average tensile strength (MPa) & 19 \\
\hline Young's modulus (GPa) & 67 \\
\hline Bulk modulus (GPa) & 41 \\
\hline Shear modulus $(\mathrm{GPa})$ & 27 \\
\hline Seismic wave velocity (m / s) & 5790 \\
\hline
\end{tabular}

Table (3) Rock properties used in the analysis $[11,12]$

\begin{tabular}{|l|c|c|c|c|c|c|c|}
\hline Rock Type & $\begin{array}{c}\text { Density, } \gamma \\
\mathbf{t} / \mathbf{m}^{\mathbf{3}}\end{array}$ & $\begin{array}{c}\text { Modulus of } \\
\text { elasticity, E } \\
\text { Gpa }\end{array}$ & $\begin{array}{c}\text { Poisson } \\
\text { ratio, } \mathbf{v}\end{array}$ & $\begin{array}{c}\text { Bulk } \\
\text { Modulus, } \\
\text { Gpa }\end{array}$ & $\begin{array}{c}\text { Shear } \\
\text { Modulus, } \\
\text { G } \\
\text { Gpa }\end{array}$ & $\begin{array}{c}\text { Unconfined } \\
\text { Compressive } \\
\text { Strength } \\
\text { Mpa }\end{array}$ & $\begin{array}{c}\text { Failure } \\
\text { Strain }\end{array}$ \\
\hline \hline Hard & 2.75 & 70 & 0.23 & 43.21 & 28.45 & 100 & 0.0025 \\
\hline Moderate & 2.4 & 30 & 0.25 & 20 & 12 & 25 & 0.005 \\
\hline Soft & 2.21 & 8.5 & 0.3 & 7.083 & 3.27 & 10 & 0.0075 \\
\hline
\end{tabular}

Table (4) General characteristics of General Purpose bombs [1]

\begin{tabular}{|l|c|c|c|}
\hline Characteristics & MK-82 & MK-84 & M-118 \\
\hline \hline Type & GP 500 bomb & GP 2000 bomb & GP 3000 bomb \\
\hline Total weight, kg & 240.9 & 894.5 & 1370 \\
\hline Charge weight, kg & $87.09 \mathrm{C} 4$ & $431.8 \mathrm{C} 4$ & $668.8 \mathrm{C} 4$ \\
\hline Case weight, kg & 141.1 & 419.6 & 408.2 \\
\hline Assembled length, cm & 228.6 & 391.2 & 464.8 \\
\hline Case diameter, cm & 27.43 & 45.72 & 61.21 \\
\hline
\end{tabular}


Proceedings of the 6 th ICCAE Conf. 16-18 May, 2006

\begin{tabular}{|l|l|}
\hline SAD4 & 46 \\
\hline
\end{tabular}

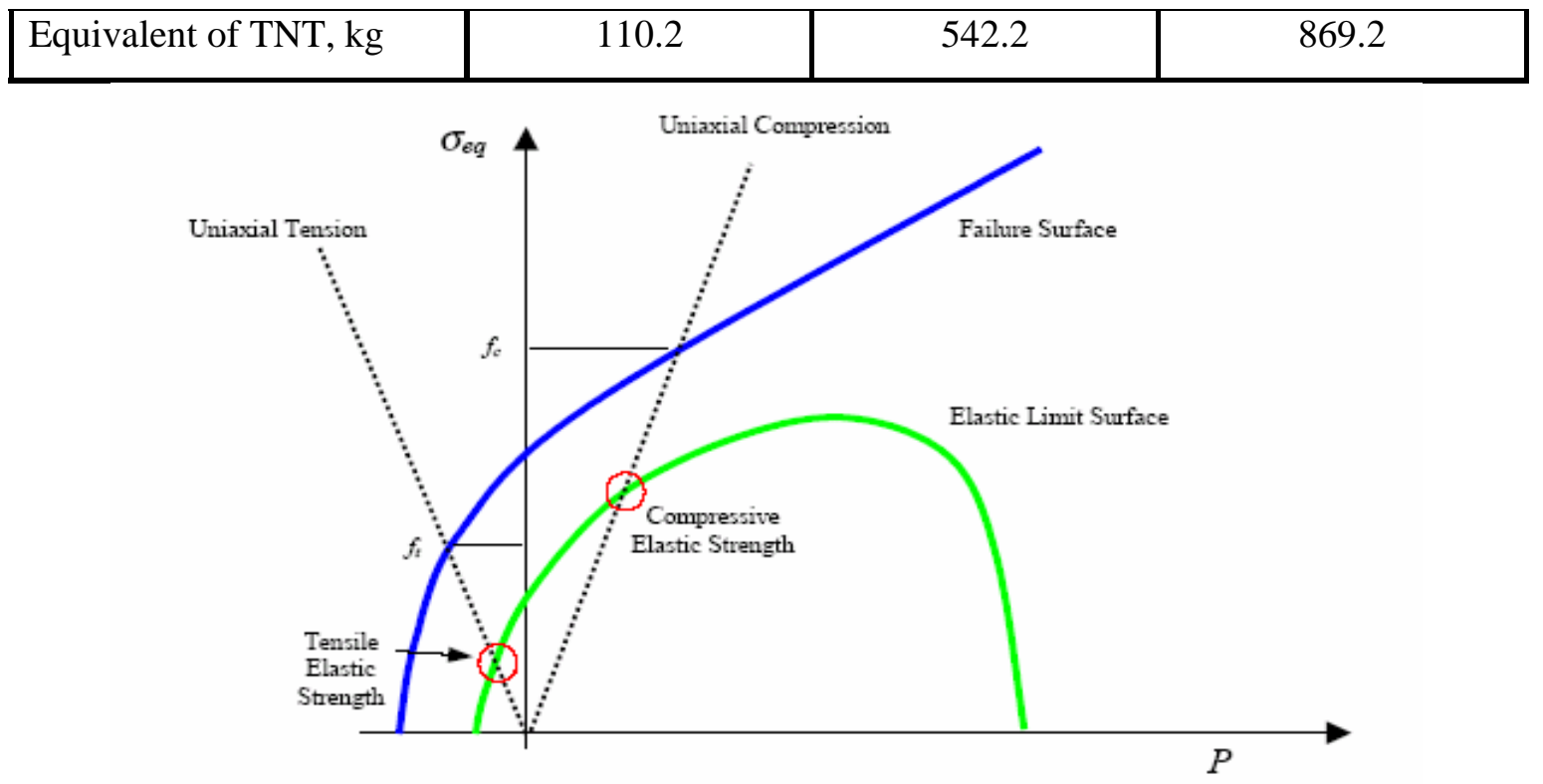

Fig. (1)- Three strength surfaces in RHT constitutive model 
Proceedings of the 6th ICCAE Conf. 16-18 May, 2006

Ground surface

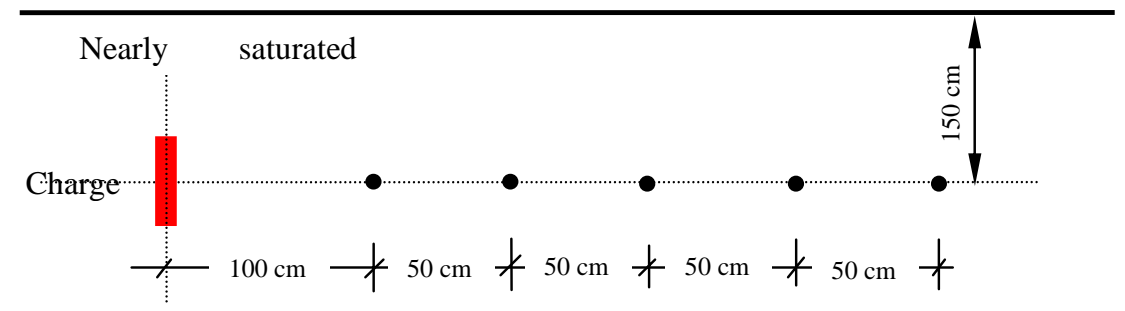

Fig. (2)- Cross section through filed test1 (not to scale) [7].

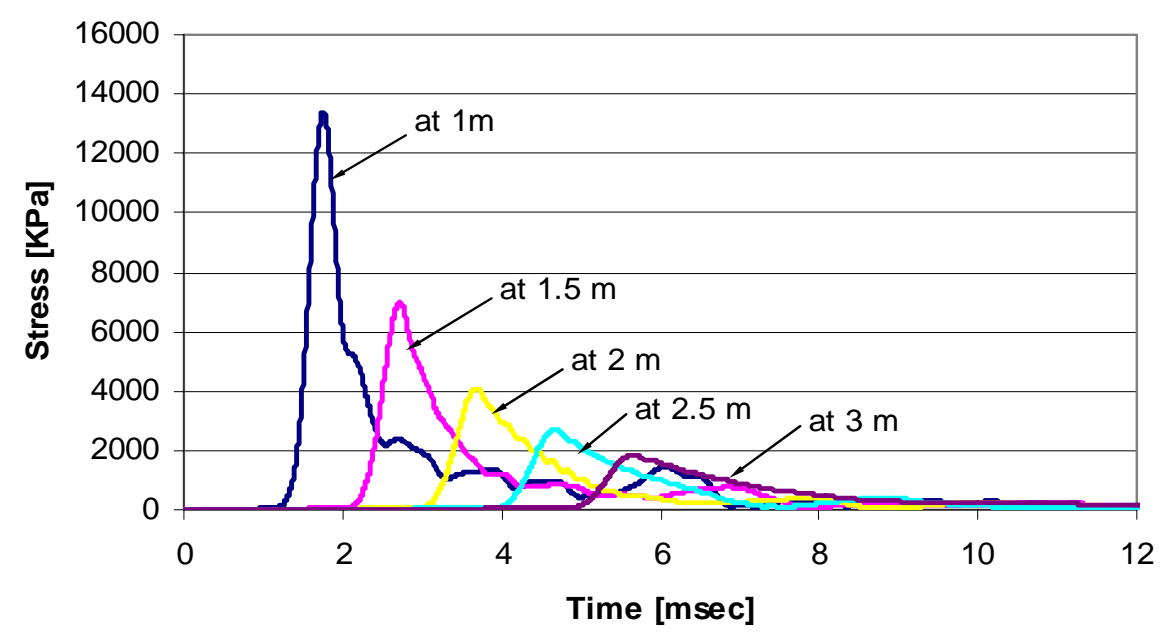

Fig. (3)- Decaying of stress time history with distance and time in nearly saturated clay at depth $1.5 \mathrm{~m}$ (charge weight $7 \mathrm{~kg}$ C4).

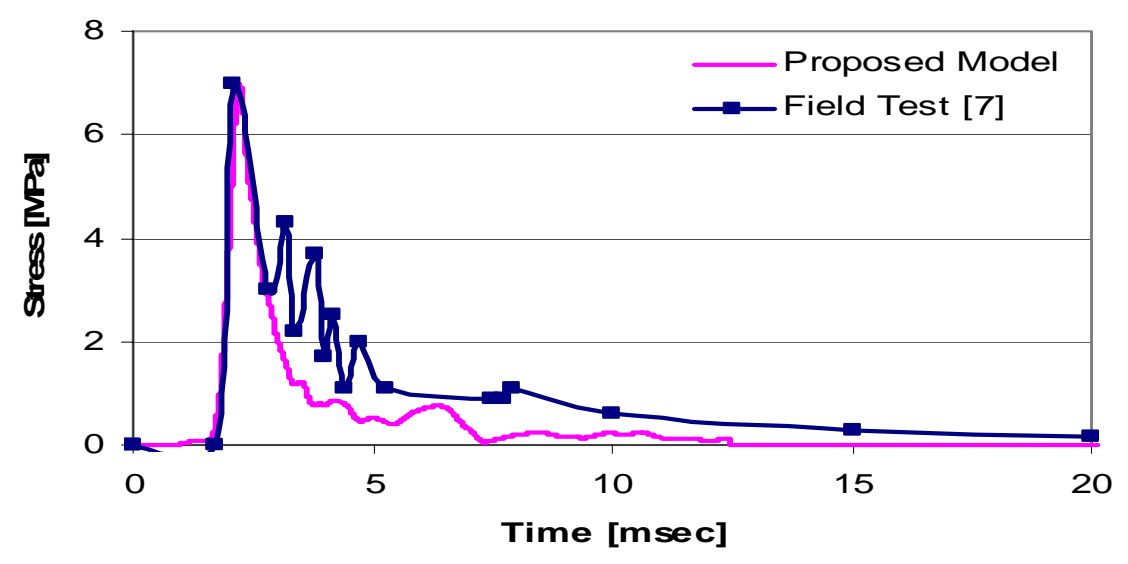

Fig. (4)- Comparison of stress at $1.5 \mathrm{~m}$ from the charge center. 


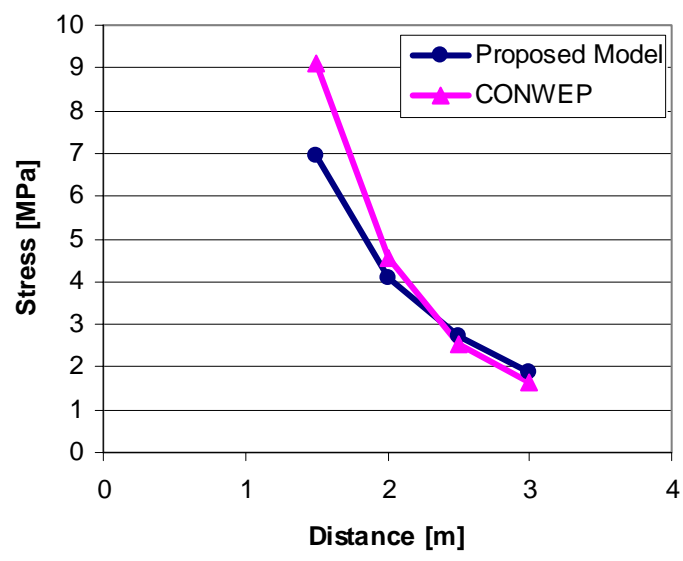

Peak stresses at depth $1.5 \mathrm{~m}$ versus horizontal distance.

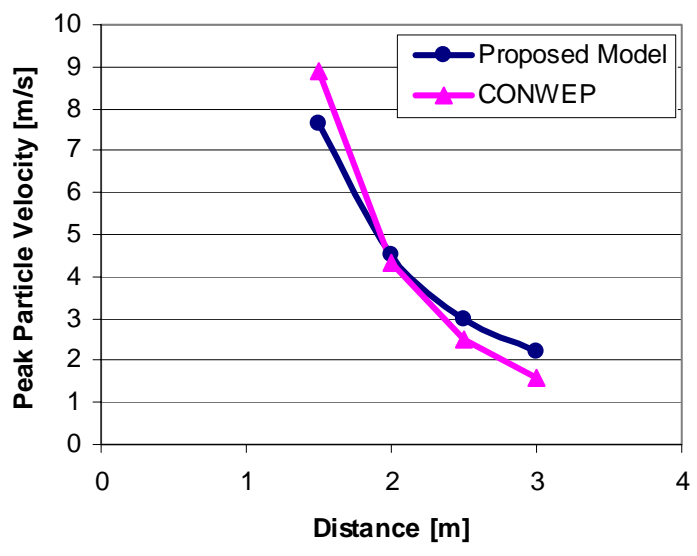

Peak Particle velocities at depth $1.5 \mathrm{~m}$ versus horizontal distance.

Fig. (5)- Comparison of obtained numerical results with corresponding CONWEP results in nearly saturated clay.

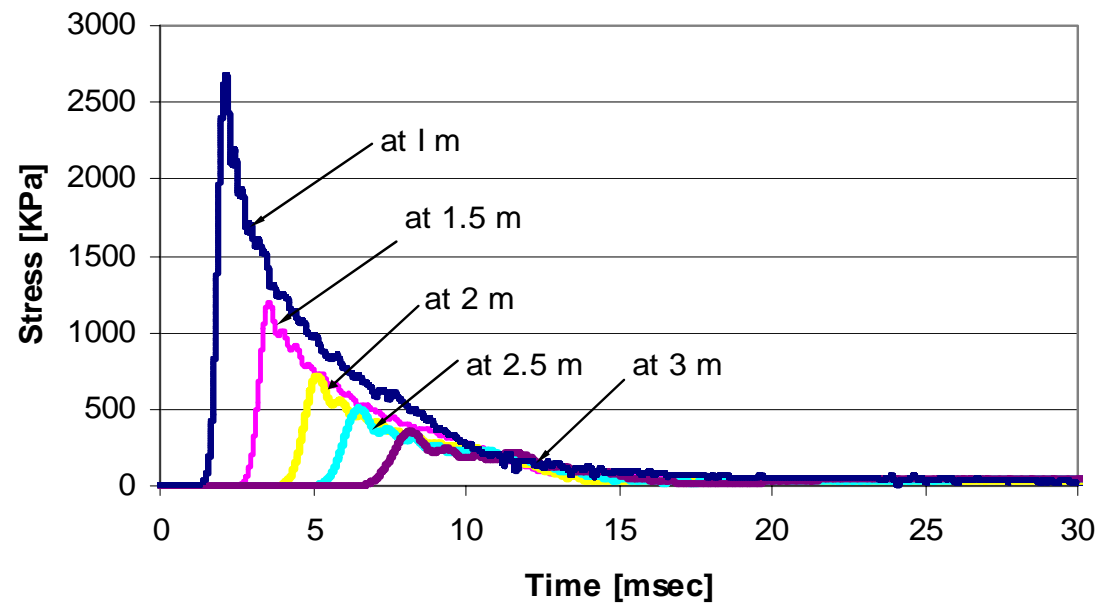

Fig. (6)- Decaying of pressure time history with distance and time at depth $1.5 \mathrm{~m}$ in sandy site (charge weight $7 \mathrm{~kg} \mathrm{C4}$ ). 


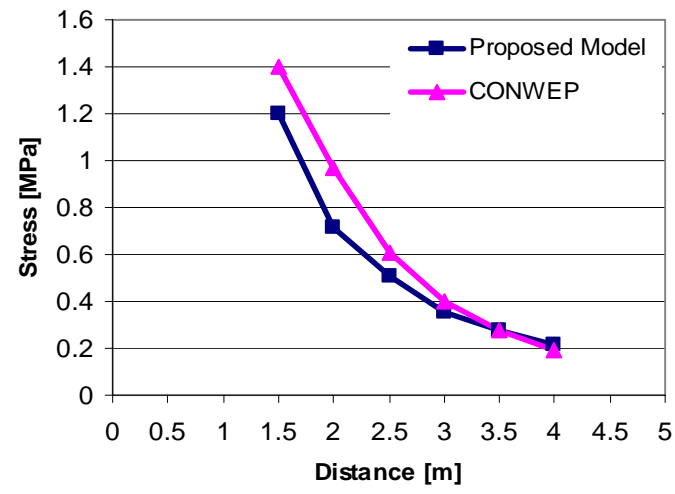

(a) Peak stresses at depth $1.5 \mathrm{~m}$ versus horizontal distance.

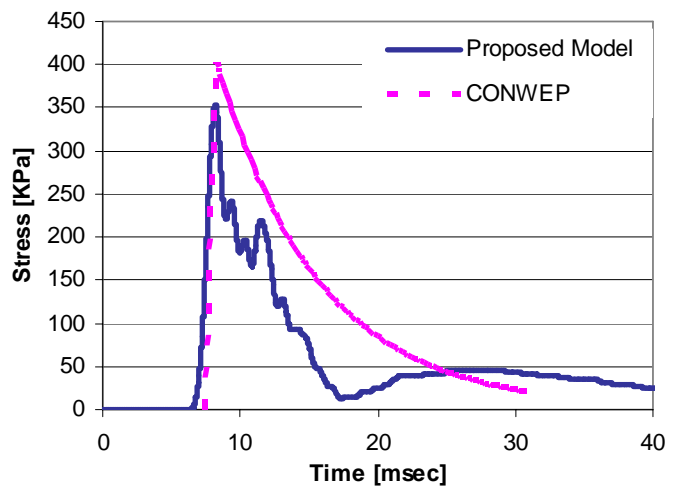

(b) Stress time histories at depth $1.5 \mathrm{~m}$ and $3 \mathrm{~m}$ horizontal range.

Fig. (7)- Comparison of obtained numerical results with corresponding CONWEP results in sandy medium.

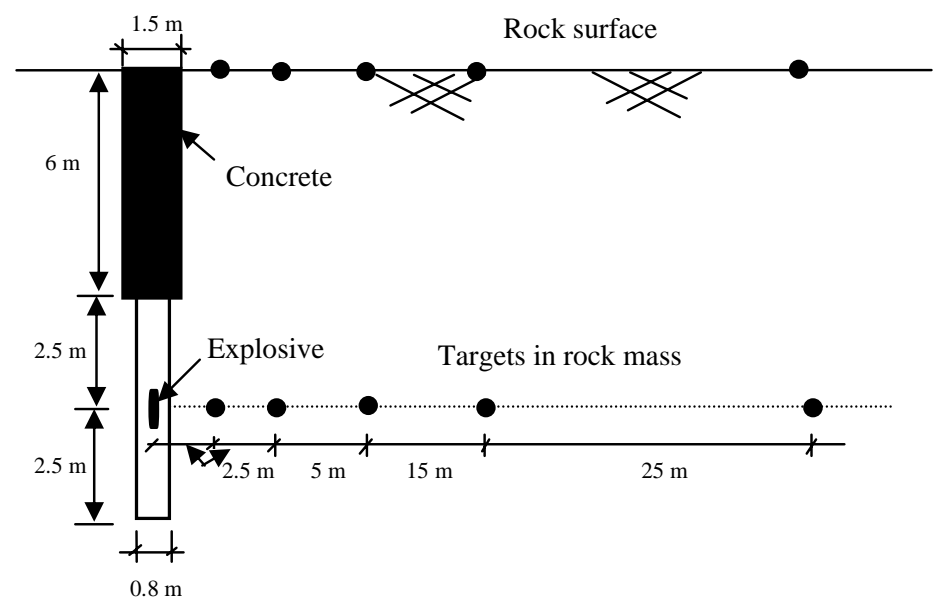

Fig. (8)- Cross section through filed test 2 (not to scale) [9]. 

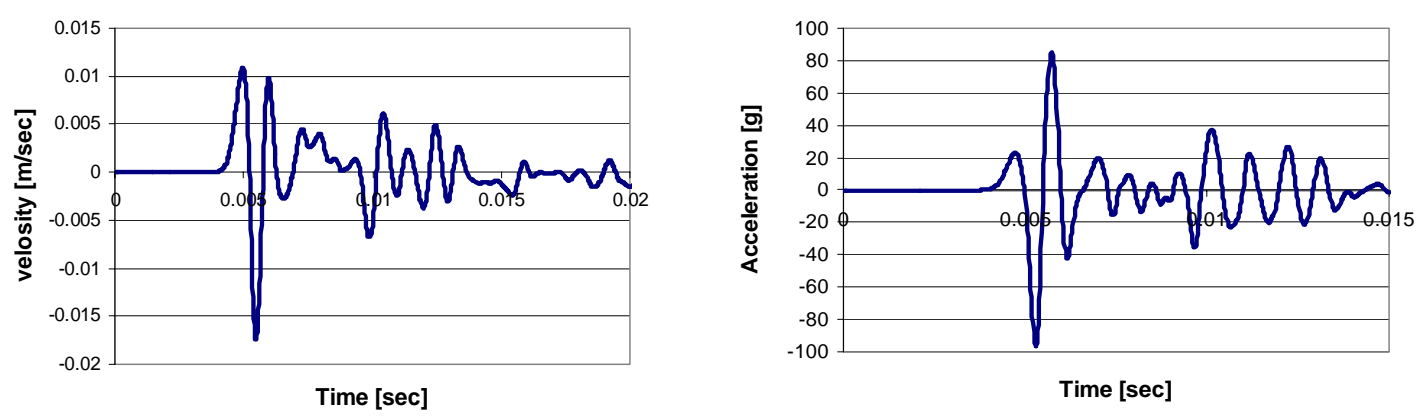

Fig (9)- Velocity and acceleration time histories in the rock mass at $25 \mathrm{~m}$ from Charge hole in the Y direction (charge weight $50 \mathrm{~kg}$ ) obtained by the proposed model.

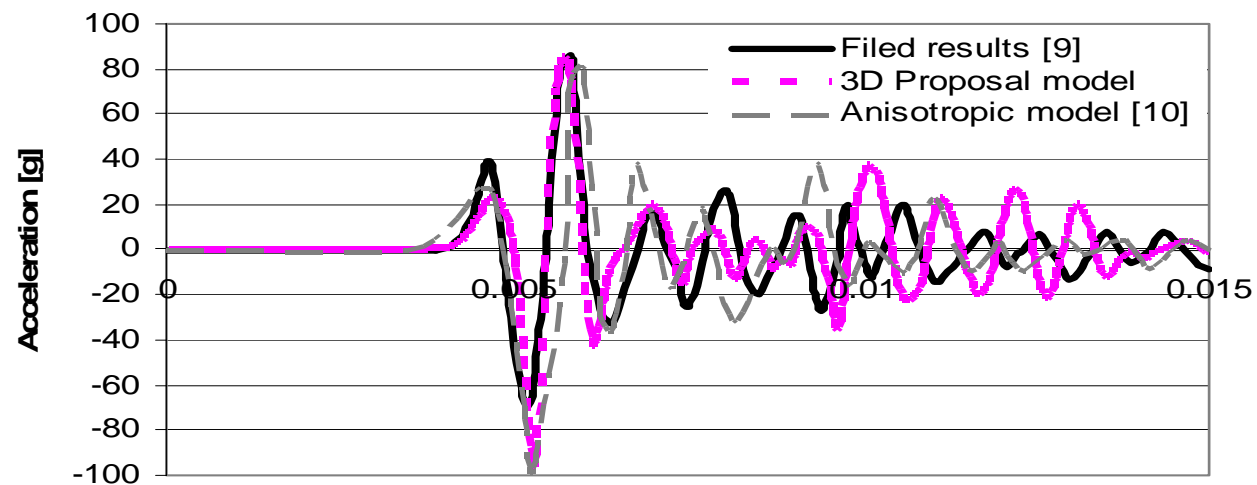

Time [sec]

Fig (10)- Comparison of acceleration time histories in the rock mass at $25 \mathrm{~m}$ from charge hole in the Y direction (charge weight $50 \mathrm{~kg}$ ).

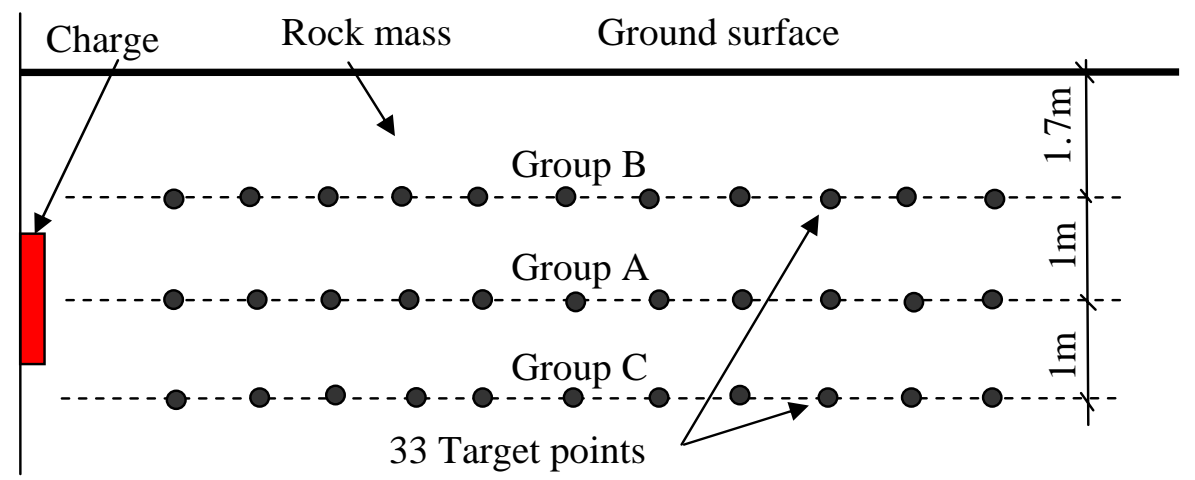

Fig. (11)- Arrangement of target points in rock mass. 


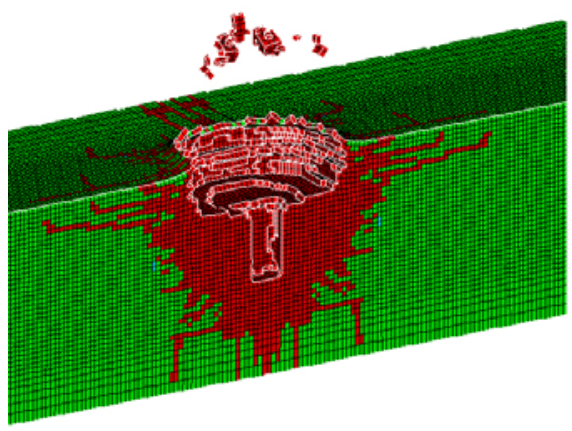

Hard

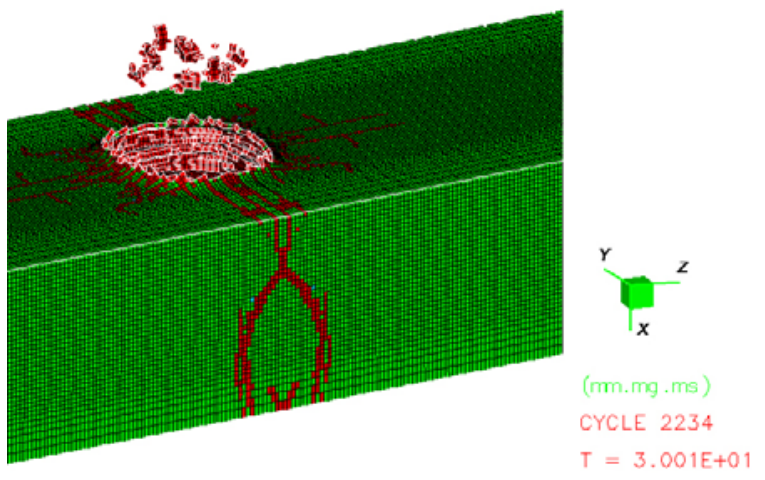

Hard

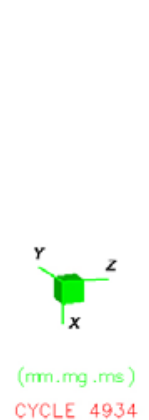

$T=3.000 \mathrm{E}+0$

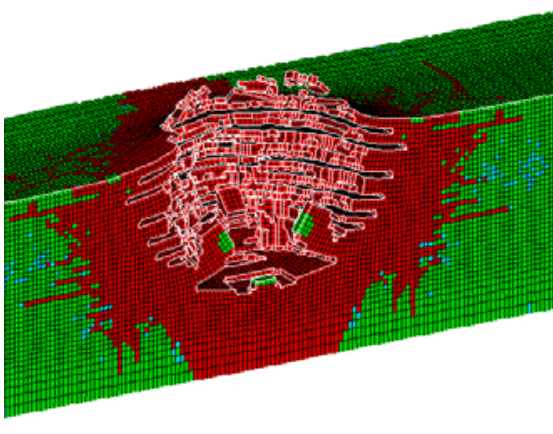

Soft

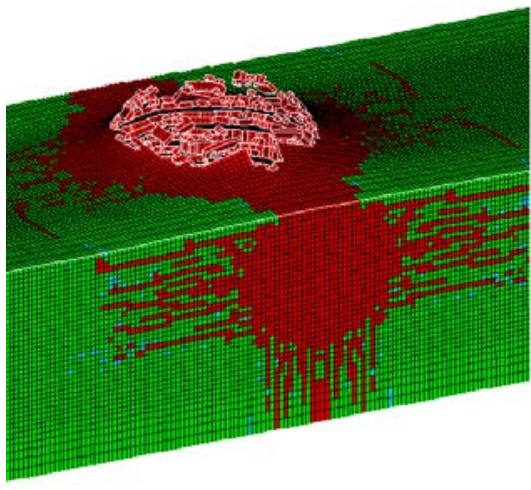

Soft

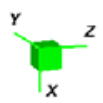

( $\mathrm{mm} \cdot \mathrm{mg} \cdot \mathrm{ms})$

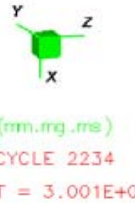

$T=3.001 E+01$

Fig. (12)- Crater formulation and damage zone in rock mediums at $30 \mathrm{msec}$ (charge weight is $542.2 \mathrm{~kg}$ of TNT, depth of burial is $1.7 \mathrm{~m}$ ).

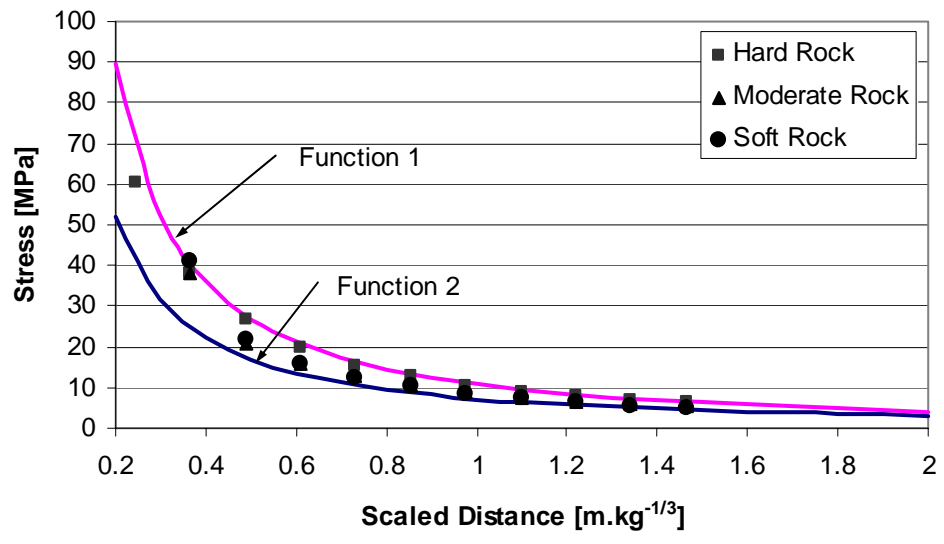

Group A 


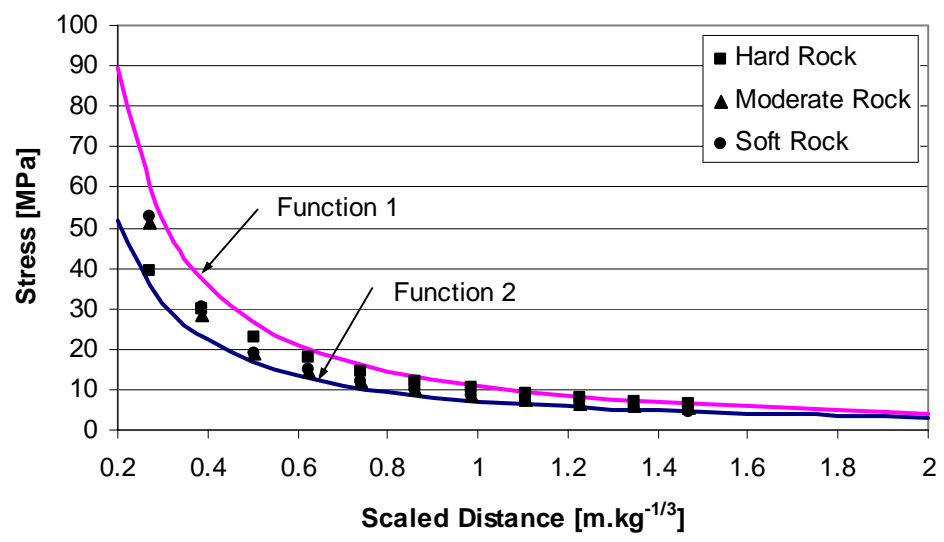

Group-B

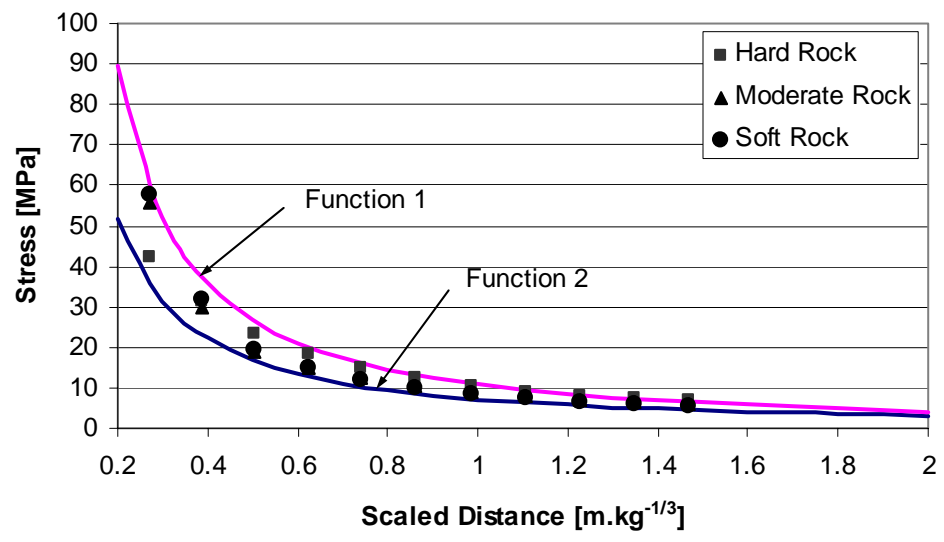

Group-C

Fig. (13)- Attenuation of stress with scaled distance.

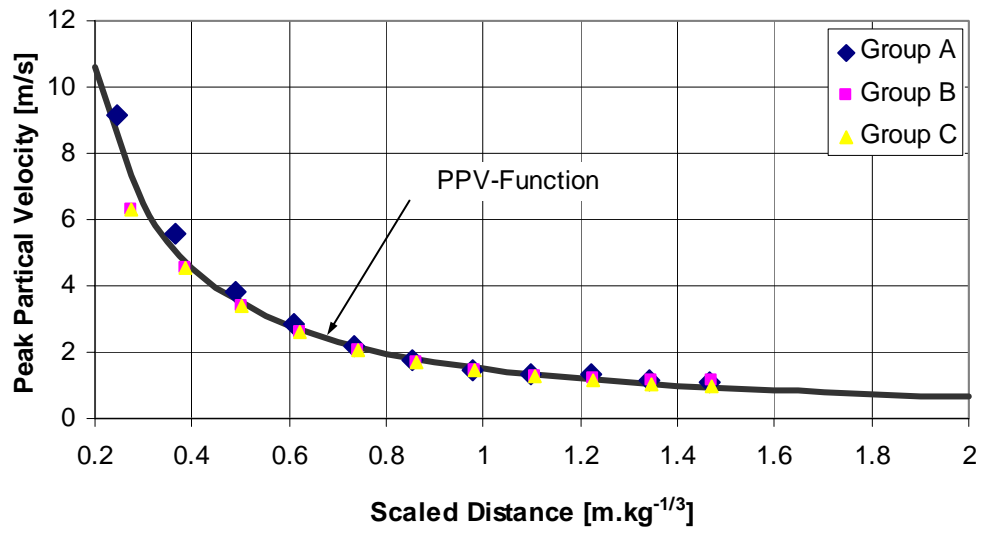

Hard Rock 


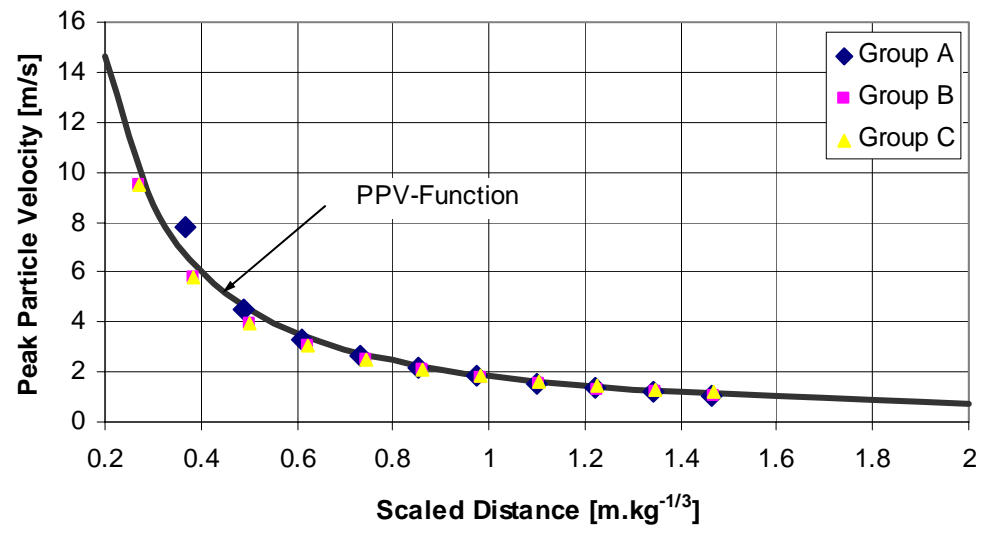

Moderate Rock

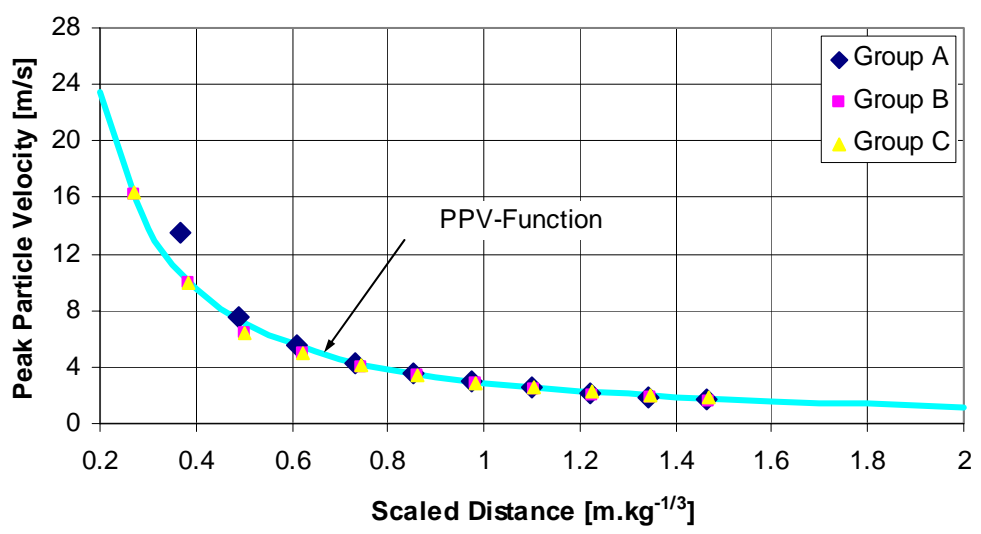

Soft Rock

Fig. (14)- Attenuation of peak particle velocity with scaled distance. 